\title{
Efecto de la Evolución del Vórtice sobre los Parámetros Hidrodinámicos de un Sistema de Dispersión Mecánica de Pigmentos
}

\author{
Jorge Ramírez-Muñoz ${ }^{(1)}$, Daniel García-Cortés ${ }^{(2)}$, José A. Colín-Luna ${ }^{(1)}$ y Carlos R. Tapia-Medina ${ }^{(1)}$ \\ (1) Departamento de Energía, Universidad Autónoma Metropolitana-Azcapotzalco. Av. San Pablo 180, \\ Col. Reynosa Tamaulipas, Azcapotzalco, D.F. 02200, México. (e-mail: jrm@correo.azc.uam.mx; \\ jacolinluna@gmail.com; ctapia64@yahoo.com.mx) \\ (2) Departamento de Medio Ambiente, Instituto Superior de Tecnologías y Ciencias Aplicadas, Avenida \\ Salvador Allende, esq. Luaces, La Habana, 10400, Cuba. (e-mail: cortes@instec.cu)
}

Recibido Nov. 13, 2015; Aceptado Ene. 14, 2016; Versión final Feb. 4, 2016, Publicado Ago. 2016

\begin{abstract}
Resumen
Se realizó una investigación numérica a fin de capturar la formación del vórtice y los parámetros hidrodinámicos (potencia, bombeo, torque, campo de velocidad y disipación viscosa) inducidos por un impulsor de alto corte tipo Hockmeyer ${ }^{\circledR}$ usando un fluido newtoniano (disolución de glucosa). El estudio se realizó en un tanque cilíndrico sin deflectores a números de Reynolds menores a 138, usando FLUENT ${ }^{\circledR}$. La formación del vórtice se simuló empleando el modelo multifásico volumen de fluido (VOF) con un enfoque de marcos de referencia múltiples. En el marco de referencia móvil (región que encierra el impulsor) se utilizó un modelo de transición a turbulento y en el volumen restante un modelo laminar. Se usaron mediciones de potencia para validar las simulaciones. Se encontró que el modelo VOF reproduce en forma aceptable el vórtice alrededor del eje. El campo de velocidad obtenido mostró dos lazos de circulación, uno por arriba y otro por debajo del impulsor, lo cual es característico de impulsores de flujo radial.
\end{abstract}

\section{Effect of the Vortex Evolution on the Hydrodynamic Parameters of a Mechanical Pigment Dispersion System}

\begin{abstract}
A numerical study has been done to capture the vortex formation and the hydrodynamic parameters (power draw, pumping, torque, field velocity and viscous dissipation) produced by a High Shear Impeller of two rings Hockmeyer ${ }^{\circledR}$ type by using a Newtonian fluid (glucose solution). The study was carried out at low Reynolds numbers $(\operatorname{Re}<138)$ in an unbaffled cylindrical tank by using FLUENT ${ }^{\circledR}$. The multiphase Volume of Fluid model (VOF) was used to capture the vortex formation together with the multiple reference frame approach. In this way, a transition model from laminar to turbulent regimen can be used in the rotation reference frame (the region enclosing the impeller) and in the remaining volume the laminar model can be used. Experimental measurements of power draw were used to validate the computational results. It was found that the VOF model acceptably predicts the vortex formation in the region near the shaft. The velocity field shows two circulation loops above and below the impeller center, which is characteristic of radial flow impellers.
\end{abstract}

Keywords: hydrodynamic parameters; pigment dispersion system; high shear impellers; unbaffled tank; viscous dissipation 


\section{INTRODUCCIÓN}

Los impulsores de alto corte (IAC) son un tipo de impulsor (agitador) que comúnmente se colocan al final de un eje y se hacen girar a velocidades angulares relativamente altas, esto crea una zona de flujo intenso y alto corte en la región cercana al impulsor. Los IAC son comúnmente usados en las industrias de pinturas, papel, tintas, cosméticos, farmacéuticas, entre otras, para incorporar pigmentos en polvo en un medio líquido y romper aglomerados de partículas con el fin de alcanzar una distribución uniforme de tamaños de partículas en el sistema. En este tipo de aplicaciones se requiere que los aglomerados de partículas se reduzcan al menor tamaño posible para alcanzar el máximo desempeño del producto final. Por ejemplo, el dióxido de titanio $\left(\mathrm{TiO}_{2}\right)$ en polvo, utilizado como un pigmento para dar brillo, cubriente, tonalidad y blancura a una gran variedad de productos tiene un tamaño de partícula primario de 0.25 micras. Sin embargo, puede existir como un polvo con aglomerados de 10 a 100 micras debido a la existencia de diversas fuerzas cohesivas, entre las que se destacan las fuerzas atractivas tipo Van der Waals (Baldyga et al., 2008). La desaglomeración solo se consigue mediante el suministro de un esfuerzo local intenso (Xie et al., 2007), en un proceso denominado dispersión que comúnmente se lleva a cabo en tanques agitados mecánicamente usando IAC.

En la industria de recubrimientos es bien conocido que en la fabricación de pinturas con altas concentraciones de pigmentos, una dispersión óptima del pigmento reduce significativamente la cantidad a usar de éste, sin afectar las propiedades ópticas de la pintura, i.e., se reduce el costo de formulación de la pintura. Con este fin, los IAC son usados en la industria de pinturas en tanques cilíndricos sin deflectores, operando en el régimen de flujo laminar y de transición a turbulento, esto último para crear un pequeño vórtice en la superficie libre, útil para succionar rápidamente los pigmentos en polvo adicionados en la superficie del fluido hacia la zona de alto corte del impulsor (Hockmeyer, 2010). Este régimen de flujo es útil para inducir los gradientes de velocidad máximos en la zona de barrido del impulsor (Ramírez-Gómez et al., 2014). A pesar de ello, en comparación con los impulsores comunes (impulsores radiales, axiales o de flujo mixto), el campo de flujo y la forma del vórtice producida por los impulsores de alto corte han sido poco estudiados usando el enfoque de la Dinámica de Fluidos Computacional (CFD, por sus siglas del inglés, Computational Fluid Dynamics). La mayor parte de la información existente en la literatura son datos técnicos proporcionados por los proveedores de estos dispositivos.

Los impulsores convencionales recomendados para el régimen de flujo de transición o turbulento (turbina Rushton, impulsores de paletas inclinadas y de alta eficiencia), son mezcladores eficientes; sin embargo, no pueden generar un mezclado local suficientemente intenso, i.e., no producen suficiente esfuerzo local de corte (cizallamiento) que favorezca la desaglomeración de partículas o polvos en un líquido, independientemente del tiempo que se mantenga la agitación (Hockmeyer, 2010). Por ello, para la desaglomeración de partículas se usan los IAC en forma de disco los cuales giran a altas velocidades y consumen muy poca energía.

En la revisión de la literatura se encontró que, exceptuando los artículos publicados por el mismo grupo de investigación autores de este trabajo (Ramírez-Gómez et al., 2014; Martínez de Jesús et al., 2014; RamírezGómez et al., 2015), en la mayoría de los estudios numéricos sobre tanques agitados sin deflectores, operando en el régimen de flujo laminar o de transición, se considera que no existe (o puede despreciarse) el vórtice que se forma sobre la superficie libre del fluido, por lo cual se supone una condición de libre deslizamiento (i.e., se desprecia la transferencia de momento) sobre toda la interfase gas-líquido (véase Lamberto et al., 1999 y Zalc et al., 2001, y referencias citadas en estos trabajos). Por otro lado, en procesos de dispersión de pigmentos, el modelado de la formación de un vórtice, aunque sea pequeño, podría ser útil para caracterizar las condiciones de operación (e.g., velocidad de agitación, viscosidad y densidad del fluido) requeridas para que los pigmentos que flotan puedan ser fácilmente incorporados hacia el seno del fluido. Más aún, caracterizar las condiciones de operación adecuadas para que el vórtice penetre hasta la zona de barrido del impulsor, manteniendo el régimen de flujo laminar (comúnmente denominado flujo de dona), permite maximizar los esfuerzos tangenciales de corte, i.e., la disipación de energía viscosa local, sobre la zona de barrido del IAC, lo cual puede influir de manera positiva en los procesos de dispersión de pigmentos (Ramírez-Gómez et al., 2015).

De acuerdo con el conocimiento de los autores, no existe un trabajo previo en la literatura en donde se aborde un estudio sobre la evolución de la forma del vórtice, y su efecto sobre los parámetros hidrodinámicos claves que influyen en los procesos de dispersión, como la disipación viscosa por unidad de masa (o unidad de volumen), para un disco impulsor de alto corte operando en el régimen de flujo evaluado en este estudio. Con este fin, en este trabajo se simula la evolución transitoria de la formación del vórtice sobre la superficie libre del fluido y se analiza su efecto en la disipación viscosa en el volumen barrido por el impulsor. 


\section{METODOLOGÍA}

\section{Material y equipo experimental}

Las mediciones de potencia para validar los resultados de las simulaciones fueron realizados en un tanque cilíndrico enchaquetado, sin deflectores, de acero inoxidable, con fondo convexo y diámetro interno $T=132 \mathrm{~mm}$ (Figura 1). Las relaciones geométricas usadas en los experimentos de distancia del impulsor al fondo del tanque $(C)$, altura del líquido $(Z)$ y diámetro del impulsor $(D)$ fueron: $C / T=0.3848, Z / T=1$ y $D / T=0.3848$, respectivamente. El equipo de agitación usado fue el Dispermat® tipo AE01 de $0.75 \mathrm{HP}$. En este equipo se midieron previamente las pérdidas por fricción asociadas con las fricciones de los rodamientos, sistema de ventilación, sellos mecánicos, etc., a través de la medición de la potencia inyectada en aire ambiental, y de esta forma se calcula automáticamente, por un algoritmo interno incluido en el equipo, la potencia neta transferida al fluido. Los datos de potencia pueden transferirse en línea hacia un ordenador por medio de una tarjeta RS232 o leerse directamente en pantalla.

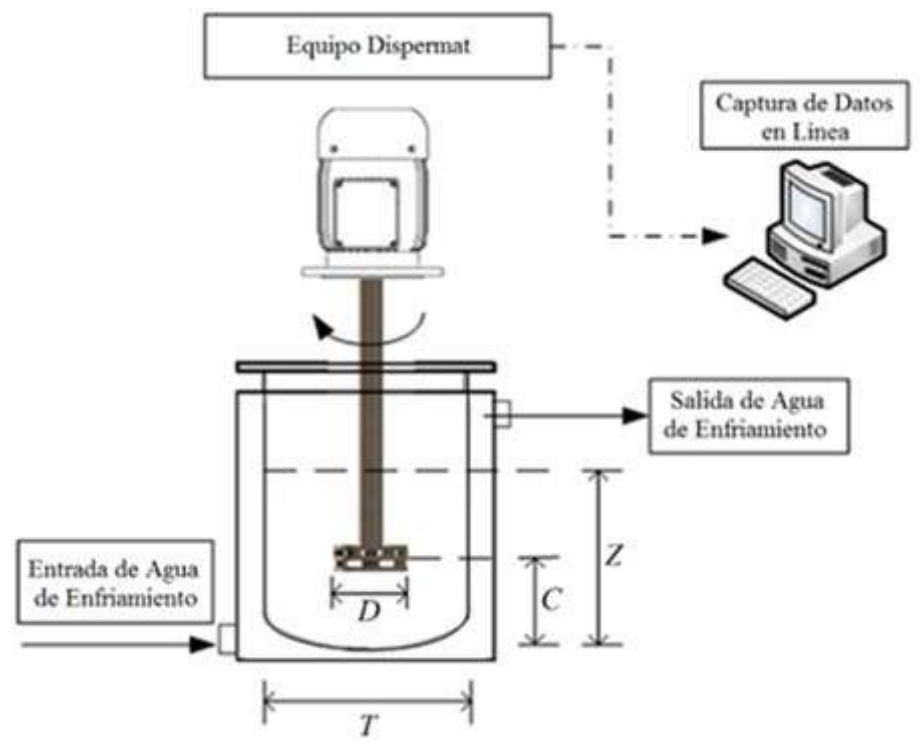

Fig. 1: Esquema del equipo experimental usado en las mediciones de potencia.

En la Figura 2 se muestra el impulsor evaluado y sus dimensiones se presentan en la Tabla 1. Como se puede observar, el impulsor tipo Hockmeyer ${ }^{\circledR}$ de dos anillos (HockD-2R) cuenta con un disco central sobre el cual se encuentran colocadas seis cuchillas, igualmente espaciadas, por arriba y debajo del disco central. Es decir, en total cuenta con 12 cuchillas.
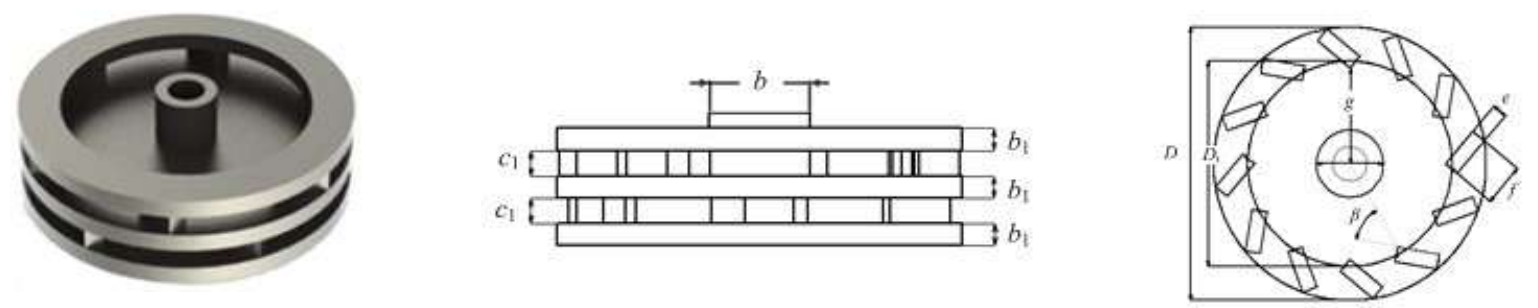

Fig. 2: Impulsor tipo Hockmeyer® de dos anillos (HockD-2R).

Tabla 1: Dimensiones del impulsor

\begin{tabular}{|c|c|}
\hline Símbolo & Dimensión $(\mathrm{mm})$ \\
\hline$b$ & 12.5 \\
\hline$b_{1}$ & 2.8 \\
\hline$c_{1}$ & 3.2 \\
\hline$D$ & 50.8 \\
\hline$D_{i}$ & 38.1 \\
\hline$e$ & 2.8 \\
\hline$f$ & 7.9 \\
\hline$g$ & 17.7 \\
\hline$\beta$ & $50^{\circ}$ \\
\hline
\end{tabular}


El fluido utilizado en el proceso de todos los experimentos y simulaciones fue una disolución de glucosa (grado alimenticio) al $36 \%$ en peso de glucosa, la cual tiene una densidad $1,376.46 \mathrm{~kg}-\mathrm{m}^{-3}$ y una viscosidad de 0.9455 $\mathrm{Pa}$-s. El valor de la viscosidad utilizada se seleccionó de forma tal que se encontrara en el intervalo de viscosidades en la cual operan comúnmente los dispersores industriales con suspensiones de pigmentos (slurries) de concentraciones entre bajas y medianas. La temperatura del fluido de proceso se mantuvo durante los experimentos a $23 \pm 0.5^{\circ} \mathrm{C}$, para ello se utilizó un baño de enfriamiento con recirculación marca Scorpion Scientific Modelo A20300 de $11 \mathrm{~L}$ de capacidad.

\section{Simulación CFD}

La simulación numérica del sistema utilizado experimentalmente se realizó aplicando el enfoque CFD. La geometría del sistema fue construida cuidando incorporar cada detalle del impulsor con el fin de simular en forma precisa, el campo de velocidades en la región cercana al impulsor y la formación del vórtice. Teniendo en cuenta que el impulsor presenta simetría radial se modeló únicamente una sexta parte del dominio y se implementó una condición de periodicidad para recuperar el dominio completo del sistema. Esta simplificación permitió reducir significativamente el número de elementos de la malla lo cual contribuyó a un ahorro del 83\% del costo de cómputo. El valor máximo de la oblicuidad admitido para una celda en todas las mallas evaluadas fue siempre menor a 0.9. En la Figura 3 se muestra la malla computacional usada la cual cuenta con 98025 celdas. Como se puede observar, el volumen se dividió en dos zonas principales, la primera que define un marco de referencia estacionario (SRF, por sus siglas del inglés Stationary Reference Frame), y la segunda que define un marco de referencia rotatorio (RRF, por sus siglas del inglés Rotating Reference Frame) la cual encierra al impulsor. Adicionalmente, en la región del RRF se delimitó un volumen que representa la zona de barrido del impulsor (VBI).

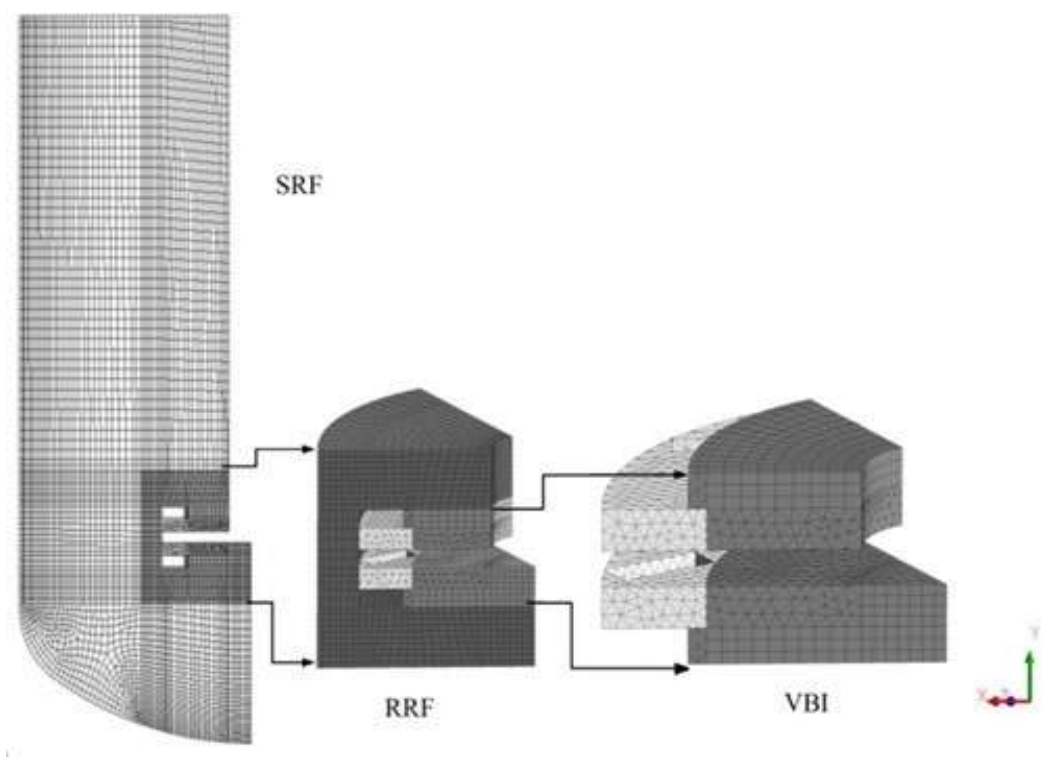

Fig. 3: Malla computacional usada en las simulaciones.

La solución numérica transitoria del sistema se obtuvo usando el software comercial de dinámica de fluidos computacional ANSYS 14.5 FLUENT. El modelo VOF se usó para simular la evolución de la interfase gaslíquido y el enfoque de marcos de referencia múltiples (MRF, por sus siglas del inglés, Multiple Reference Frame) se adoptó para modelar el movimiento del impulsor (región RRF). El modelo de transición a flujo turbulento de tres ecuaciones $\kappa-\kappa l-\omega$ se usó en algunas simulaciones para evaluar la disipación de energía turbulenta en el volumen que rota (RRF) y compararlas con la disipación de energía viscosa predicha por el modelo laminar. Dicho modelo se usó en todas las simulaciones en el resto del volumen, i.e., en el marco de referencia estacionario (SRF). Para acoplar la velocidad con la presión se implementó el esquema estándar, mientras que el esquema de discretización utilizado fue el QUICK. Se construyó una sola malla para modelar el líquido y el aire, y se empleó un modelo de fuerza de superficie continua incorporado en Fluent para modelar la transferencia de momento en todo el dominio. Atendiendo a los requerimientos del modelo VOF, se definió como fluido primario al aire y como fluido secundario la disolución de glucosa, y además se aplicó un modelo de balance de fuerzas para el transporte de momento a través de la frontera gas-líquido. Todas las simulaciones se detuvieron en $10 \mathrm{~s}$, a estos tiempos de cómputo, las soluciones se consideraron totalmente convergentes puesto que los valores de los residuos de las ecuaciones arrojaron valores aproximados a $10^{-5}$. 


\section{RESULTADOS Y DISCUSIÓN}

Los resultados se dividen en cuatro secciones, en la primera sección se presenta un análisis de independencia de malla, en la segunda sección se validan los resultados de la simulación con mediciones experimentales de potencia, en la tercera sección se presentan los resultados del campo de velocidad que induce el IAC y en la última sección se presentan los resultados de la evolución transitoria del vórtice mediante gráficos de contornos de fases (disolución de glucosa - aire) a diferentes velocidades de agitación. La región de barrido del impulsor es la zona en donde existe la mayor probabilidad que ocurra la máxima eficiencia de dispersión, puesto que es la zona en donde ocurre la mayor disipación (Ramírez-Gómez et at., 2014). Finalmente, en la última sección también se aborda una discusión de la evolución de la viscosidad y de la potencia por unidad de masa (y por unidad de volumen) en la región barrida por el impulsor.

\section{Análisis de independencia de malla}

Con la finalidad de evaluar la independencia de las predicciones numéricas de los números de potencia $\left(N_{P}\right)$ y bombeo $\left(N_{Q}\right)$ con respecto a la densidad de la malla se crearon varias mallas para cada impulsor, incrementando la densidad de celdas. El número de potencia se define como (Bird, 2006):

$$
N_{P}=\frac{P}{\rho N^{3} D^{5}},
$$

donde $P$ es la potencia neta suministrada al fluido, $\rho$ es la densidad del fluido y $N$ es la velocidad de rotación del agitador en revoluciones por segundo. La potencia se estimó de los resultados de las simulaciones a partir de la torsión mecánica $(M)$ que ejerce el fluido sobre el impulsor, i.e., $P=2 \pi N M$. Por otro lado, el número de bombeo está dado por (Paul et al., 2004):

$$
N_{Q}=\frac{Q}{N D^{3}},
$$

donde $Q$ es el flujo radial descargado por el impulsor a través de una superficie cilíndrica cuyo diámetro y altura coinciden con el diámetro y altura del impulsor.

En la Figura 4 se muestran los valores obtenidos de $N_{P}$ y $N_{Q}$ en función del número de celdas (41602, 79816, 98025, 180530 y 288821) a 1200 rpm. Nótese que para la malla de 79816 elementos, las predicciones de $N_{P}$ no cambian significativamente con un mayor refinamiento; sin embargo, si existen cambios importantes en los valores de $N_{Q}$.

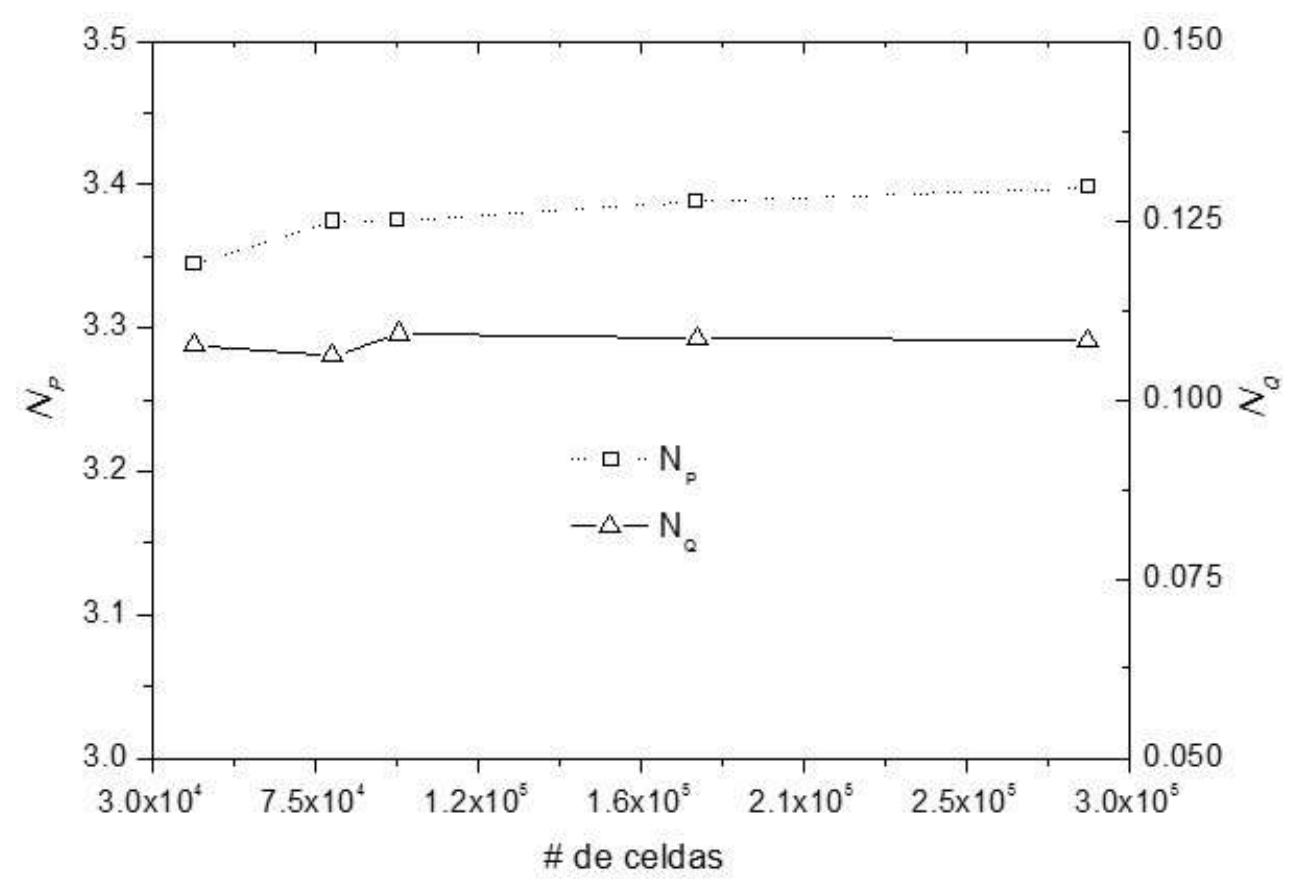

Fig. 4: Análisis de independencia de los resultados de las simulaciones con respecto a la densidad de la malla. 
De acuerdo a estos resultados, el criterio usado en este trabajo establece que sólo existe una independencia de los resultados de la simulación con respecto a la densidad de la malla cuando tanto $N_{P}$ como $N_{Q}$ no varían significativamente, de esta forma el número de celdas finalmente usado para todas las simulaciones fue de 98025 .

\section{Validación de los resultados de las simulaciones usando mediciones de potencia}

La validación de las simulaciones numéricas en tanques agitados usando los valores de número de potencia obtenidos experimentalmente es una práctica común reportada en la literatura (e.g., Werner et al., 2014). En la Figura 5 se muestran los resultados de las mediciones experimentales de consumo de potencia del impulsor HockD-2R realizadas en este trabajo y los resultados de las simulaciones. Como se puede observar, los resultados de las simulaciones son muy similares a las mediciones experimentales (con un error relativo promedio inferior al $5 \%$ ), lo cual sugiere que los resultados obtenidos aplicando el enfoque CFD son confiables.

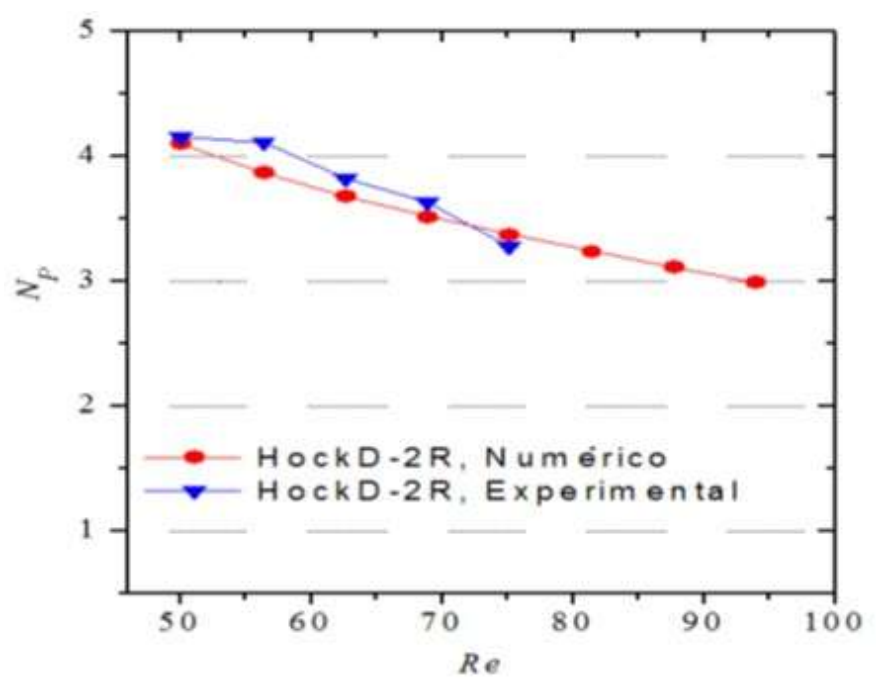

Fig. 5: Comparación del número de potencia numérico contra el experimental.

\section{Campos de velocidad}

En la Figura 6 se muestran los vectores de magnitud de la velocidad, los cuales fueron normalizados usando la velocidad periférica del impulsor $\left(V_{T i p}=\pi D N\right)$ a $1200 \mathrm{rpm}$. Estos fueron evaluados en cuatro posiciones azimutales diferentes. Las posiciones donde se evaluaron los vectores de velocidad para los dos impulsores se muestran en la parte superior de cada figura. En todos los planos se observa la existencia de dos lazos de circulación ubicados por encima y por debajo del centro del impulsor. Este patrón de flujo, en donde se forman dos zonas de recirculación separadas ha sido reportado en estudios experimentales y numéricos en tanques agitados con una turbina Rushton operando en el régimen laminar (Lamberto, 1999). Como se puede observar en la figura, el patrón de flujo inducido por el impulsor de alto corte HockD-2R es similar al reportado para la turbina Rushton, con la particularidad de que las velocidades máximas se encuentran en el espacio comprendido entre los anillos que, a su vez, corresponde con la zona que barren las cuchillas.

El patrón de flujo, en términos de las magnitudes de los vectores de velocidad normalizados con respecto a la velocidad periférica $\left(V / V_{T i p}\right)$, también fue evaluado a $1200 \mathrm{rpm}$ en cuatro planos horizontales ubicados a diferentes alturas (Figura 7). Las posiciones donde se evaluaron los vectores de velocidad se muestran en los insertos de las figuras 7a-7b. Se observa nuevamente que las zonas de mayor velocidad son las que corresponden al volumen barrido por las cuchillas, lo cual sugiere que las cuchillas del impulsor HockD-2R causan el bombeo más intenso que provoca el impulsor.

\section{Contornos de fases y parámetros hidrodinámicos en la zona de barrido del impulsor}

El efecto de la velocidad de agitación en la formación del vórtice alrededor del eje del impulsor se muestra en la Figura 8 a través del contorno de fracción volumétrica de fases obtenido a tres velocidades de agitación diferentes (1200, 1800 y 2200 rpm). El color azul representa la disolución de glucosa al $36 \%$ y el color rojo el aire, por lo cual una disminución de la intensidad del color azul significa que una cierta cantidad de aire está quedando retenida en el líquido. 


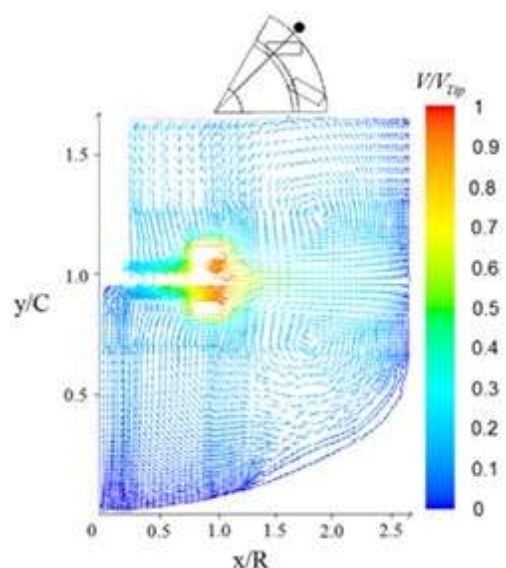

a) $15^{\circ}$

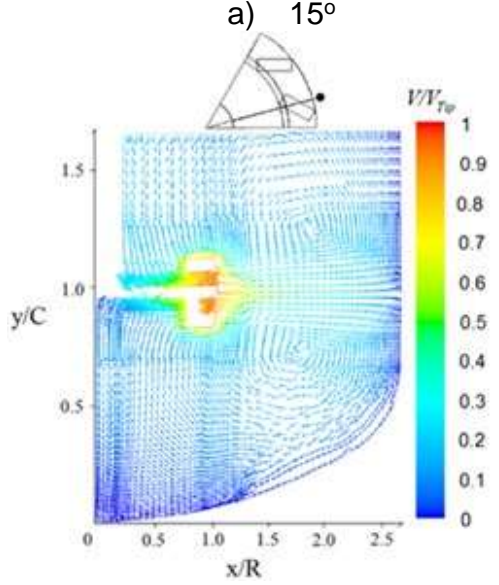

c) $45^{\circ}$

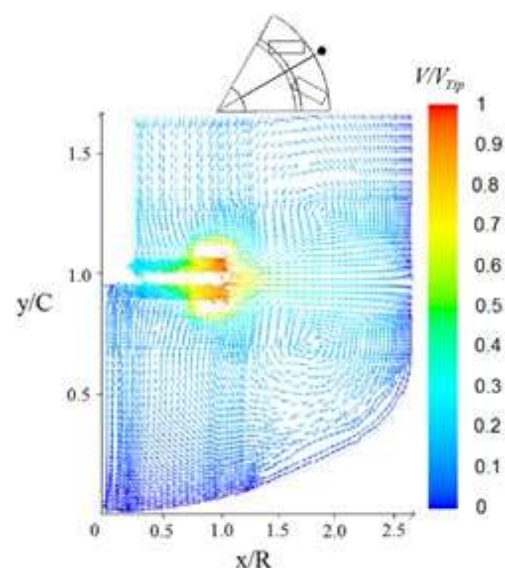

b) $30^{\circ}$

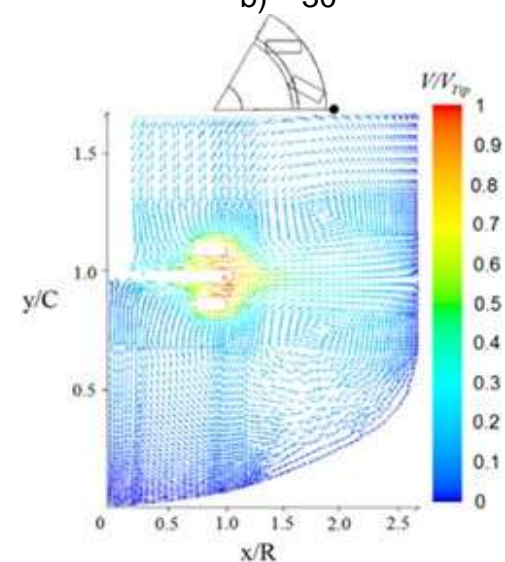

d) $60^{\circ}$

Fig. 6: Vectores de magnitud de velocidad evaluados en cuatro posiciones azimutales diferentes a $1200 \mathrm{rpm}$.

Como puede observarse, a medida que se incrementa la velocidad de agitación, la altura de la interfase gas-líquido se incrementa significativamente por la disminución de la densidad efectiva del líquido, lo cual es causado por el aire que ha quedado atrapado. Para $2200 \mathrm{rpm}$ se observa que el ancho del vórtice que penetra desde la superficie libre al disco central es de menor grosor con respecto a las demás velocidades de agitación (1200 y 1800 rpm). Los lazos de circulación observados en las Figuras 6a - 6d también pueden apreciarse en esta figura, incluso puede verse que a $2200 \mathrm{rpm}$ la región central de los lazos de circulación no se mezcla con el resto del fluido contenido en el tanque, i.e., permanece segregada, lo cual no es recomendable para procesos de dispersión de pigmentos. Hay que mencionar que una entrada excesiva de aire al sistema es indeseable en procesos de dispersión ya que disminuye también la viscosidad efectiva del líquido y puede incluso ocasionar turbulencia, lo cual es contraproducente en procesos de dispersión. Por lo tanto, los resultados obtenidos a partir de las simulaciones pueden ser útiles para caracterizar la velocidad máxima a la cual se puede operar el equipo, e incluso predecir condiciones de operación en las cuales el líquido pudiera llegar a derramarse del tanque por la entrada excesiva de aire al sistema.

En la Figura 9 se muestran los contornos de la evolución del vórtice a una velocidad de agitación de 2200 rpm durante la etapa inicial de la agitación (arranque) con intervalos de $0.16 \mathrm{~s}$. En el tiempo inicial $(0 \mathrm{~s})$ la interfase aire-líquido es prácticamente plana, posteriormente el aire penetra hasta la zona de barrido del impulsor (0.48 s), lo cual provoca que la viscosidad dinámica y el torque comiencen a disminuir (Figura 10). Puede observarse que a este mismo tiempo tanto la disipación viscosa por unidad de volumen como por unidad de masa evaluados en la zona de barrido del impulsor comienzan un incremento significativo, el cual se debe a que la presencia del vórtice incrementa los gradientes de velocidad en la zona de barrido del impulsor, y esto a su vez, provoca el aumento de la disipación viscosa (Ramírez-Gómez et al., 2015).

Hay que señalar que en las simulaciones donde se usó el modelo de transición a flujo turbulento de tres ecuaciones $\kappa-\kappa l-\omega$, los valores de la disipación de energía turbulenta en el volumen que rota (RRF) resultaron por lo menos cinco órdenes de magnitud inferiores a los de la disipación viscosa, por lo que se consideró régimen de flujo laminar en todo el volumen del reactor y la disipación viscosa fue calculada introduciendo la función de disipación viscosa en Fluent como una función definida por el usuario como se ha sugerido en otros trabajos (Ramírez-Gómez et al., 2015). 


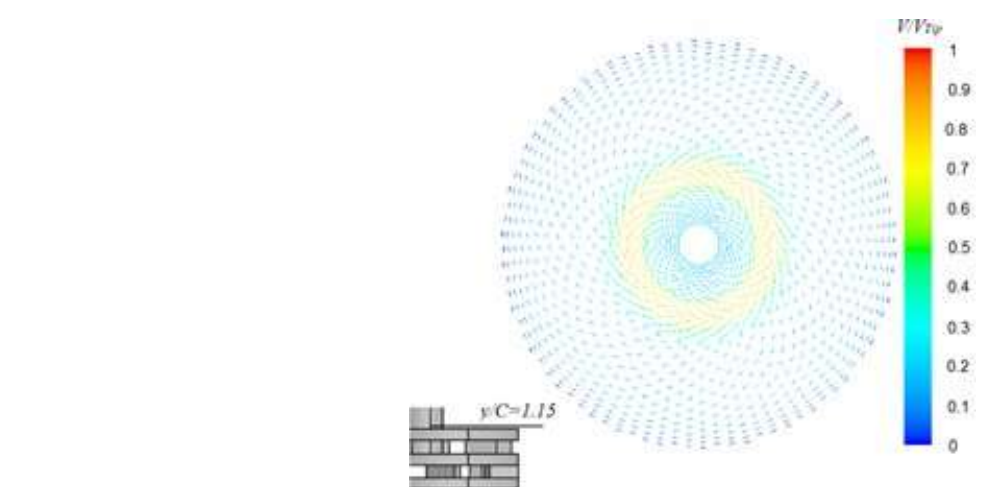

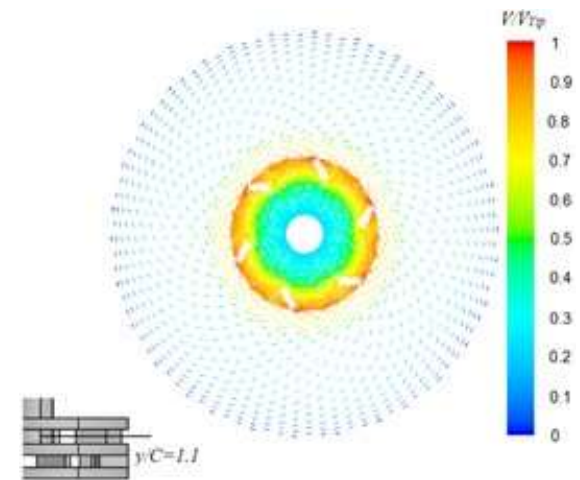

b) $y / C=1.1$.

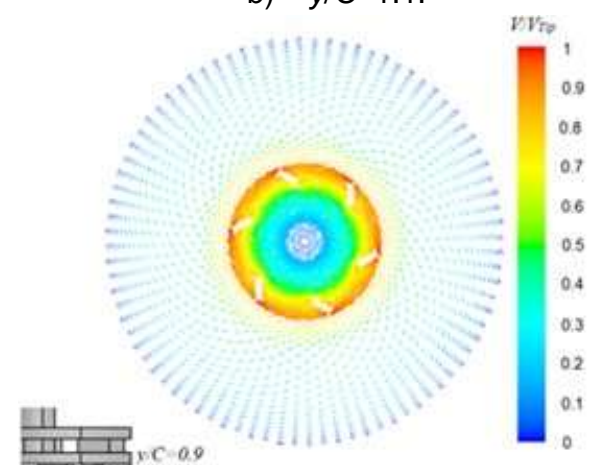

d) $y / C=0.9$.

a) $y / C=1.15$

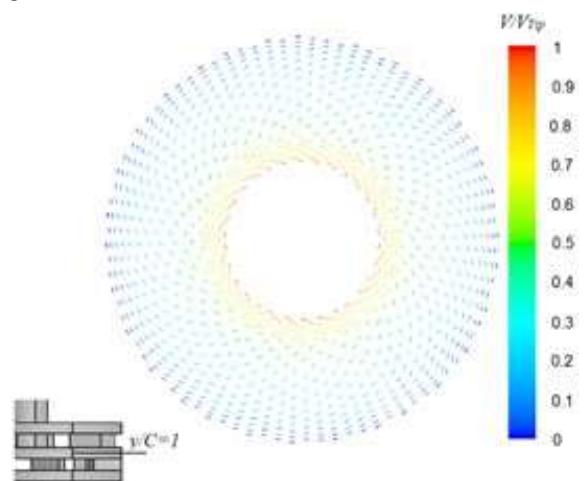

c) $y / C=1$.

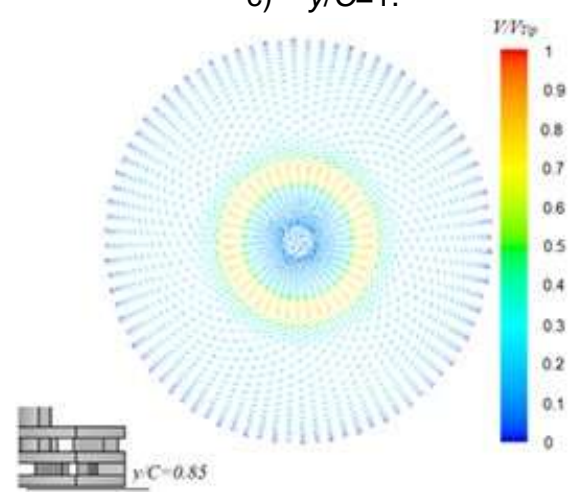

e) $y / C=0.85$

Fig. 7: Vectores de velocidad en el plano horizontal evaluados en cinco posiciones axiales a $1200 \mathrm{rpm}$.
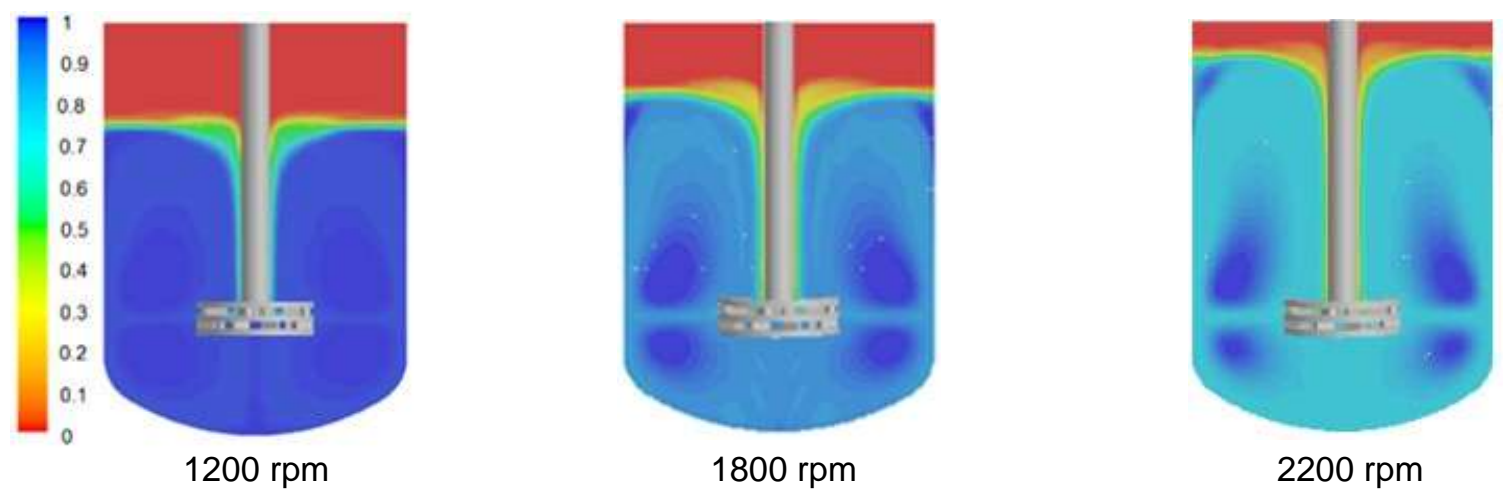

Fig. 8: Contornos de fase a tres velocidades de agitación con tiempos de simulación de $10 \mathrm{~s}$.

En la Figura 10 se observa además que la viscosidad cinemática $(v=\mu / \rho$ donde $\mu$ y $\rho$ son la viscosidad dinámica y la densidad, respectivamente) permanece constante, es decir, el efecto de la reducción de la viscosidad por la entrada del aire se compensa con la reducción de la densidad del fluido, por lo cual $v$ no cambia significativamente. También puede observarse que inicialmente la disipación viscosa por unidad de volumen es mayor que la disipación viscosa por unidad de masa, sin embargo, aproximadamente a los $5 \mathrm{~s}$ ambas relaciones se igualan y permanecen prácticamente constantes, mientras que a los $8 \mathrm{~s}$ la disipación 
viscosa por unidad de masa se hace ligeramente mayor y esta tendencia se extiende hasta los $10 \mathrm{~s}$. Considerando que el factor de conversión entre la disipación viscosa por unidad de volumen y la disipación viscosa por unidad de masa es el inverso de la densidad, este comportamiento puede asociarse a los cambios de la densidad del fluido en la zona de barrido del impulsor. De esta forma, para valores de $\rho>1000 \mathrm{~kg} \mathrm{~m}^{-3}$, la disipación viscosa por unidad de volumen será mayor a la disipación viscosa por unidad de masa, mientras que si $\rho<1000 \mathrm{~kg} \mathrm{~m}^{-3}$ la segunda es mayor que la primera, por último, si $\rho=1000 \mathrm{~kg} \mathrm{~m}^{-3}$, ambas disipaciones son idénticas.

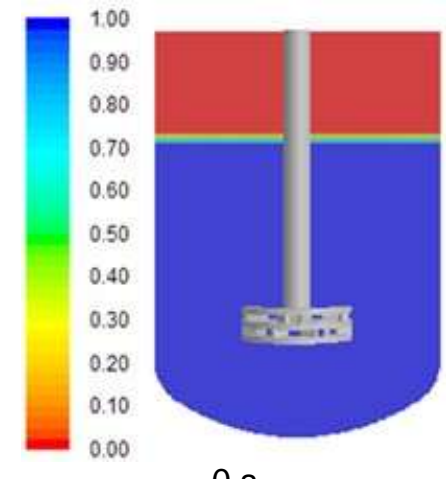

$0 \mathrm{~s}$

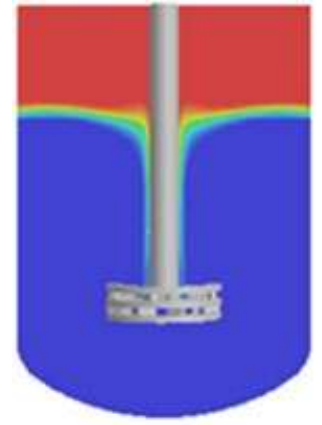

$0.48 \mathrm{~s}$

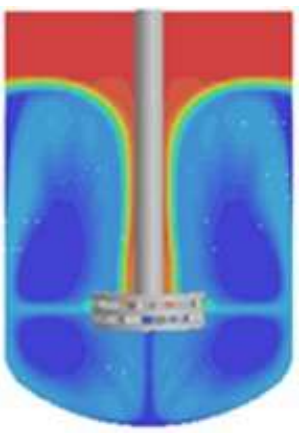

$2 s$

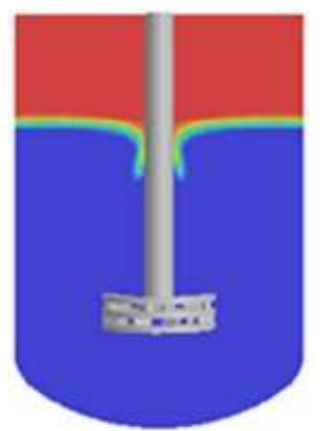

$0.16 \mathrm{~s}$

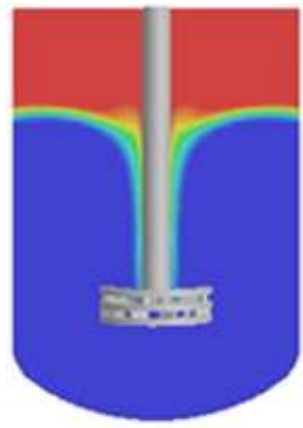

$0.60 \mathrm{~s}$

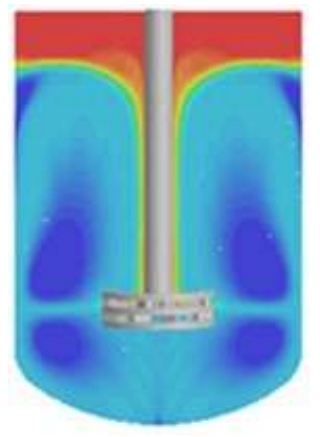

$4 \mathrm{~s}$

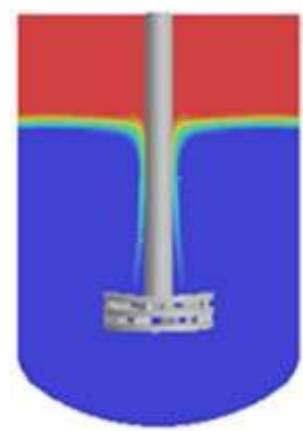

$0.32 \mathrm{~s}$

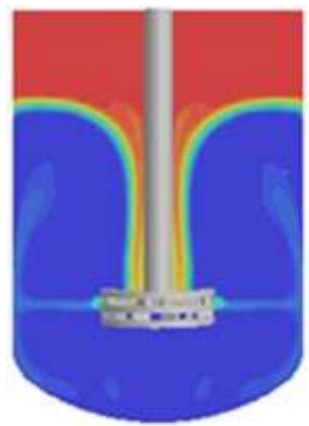

$1 \mathrm{~s}$

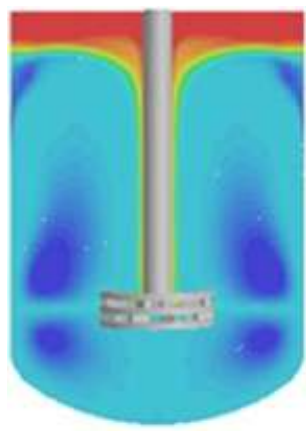

$8 \mathrm{~s}$

Fig. 9: Evolución del vórtice y de los contornos de fase a 2200 rpm.

\section{CONCLUSIONES}

El estudio muestra que es posible reproducir adecuadamente la forma del vórtice que se forma sobre la superficie libre de un tanque agitado con un disco dispersor de alto corte operando en el régimen laminar usando CFD, y además, que este enfoque puede utilizarse para determinar condiciones de operación adecuadas en procesos de dispersión de pigmentos. Los resultados obtenidos muestran que en procesos de dispersión de pigmentos con condiciones de operación similares a las evaluadas existen regiones segregadas de fluido, las cuales no se mezclan con el resto del sistema. En estas regiones segregadas, la eficiencia de dispersión de pigmentos sería inferior al resto del volumen de fluido que circula por la región de barrido del impulsor. Con el fin de eliminar estas regiones, y considerando que la existencia de un vórtice en la superficie libre del tanques resulta útil en procesos de dispersión para una rápida succión de los sólidos en polvo hacia la zona de barrido del impulsor, sería oportuno estudiar el efecto de la instalación de deflectores de altura y ancho inferior al recomendado para tanques agitados operando en régimen turbulento. En el sistema de dispersión estudiado, se encontró que la disipación viscosa por unidad de volumen en la zona de barrido del impulsor a 2200 rpm (i.e., en la zona de máxima disipación de energía) se iguala a la disipación viscosa por unidad de masa debido a la disminución de la densidad ocasionada por la entrada de aire al sistema. 


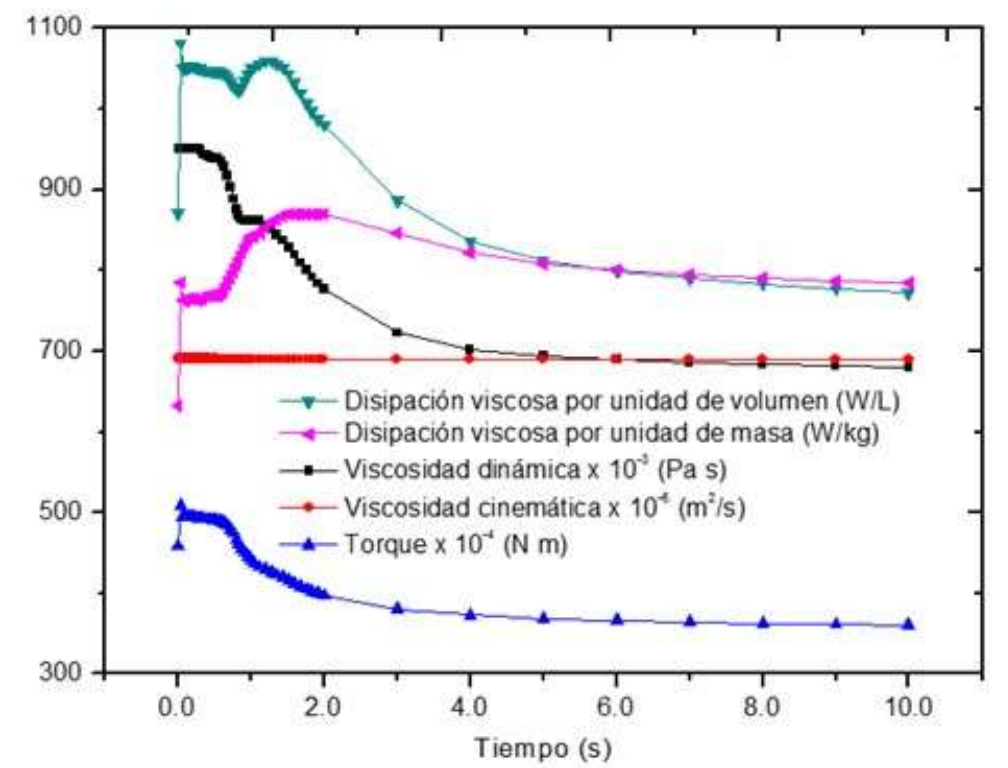

Fig. 10: Evolución de distintas variables de interés durante $10 \mathrm{~s}$ de simulación.

\section{REFERENCIAS}

Baldyga, J.; L. Makowskia; W. Orciucha; C. Sauterb y H.P. Schuchmann, Deagglomeration processes in high-shear devices, Chem. Eng. Res. Des., 8(6), 1369-1381 (2008)

Bird, R.; W. Stewart y E. Lightfoot, Fenómenos de Transporte, 2a edición, Limusa Wiley, México, D.F., México (2006)

Hockmeyer, H., Practical Guide to High-Speed Dispersion, Paint Coat. Ind.: 26, $32-36$ (2010)

Lamberto, D.J; M.M. Alvarez y F.J. Muzzio, Experimental and computational investigation of the laminar flow structure in a stirred tank, Chem. Eng. Sci.: 54, 919-942 (1999)

Martínez de Jesús, G.; R. Ramírez-Gómez; J. Ramírez-Muñoz y D. García-Cortés, Simulación CFD de la hidrodinámica inducida por un dispersor tipo Norstone, 1-12, Memorias de la 17 Convención Científica de Ingeniería y Arquitectura, La Habana, Cuba 24 al 28 de noviembre (2014)

Paul, E.L.; V.A. Atiemo-Obeng y S.M. Kresta, Handbook of Industrial Mixing, Science and Practice, John Wiley \& Sons, Inc., New Jersey, USA (2004)

Ramírez-Gómez R. y otros siete autores, Simulación CFD de la hidrodinámica inducida por un disco dispersor de alto corte tipo Hockmeyer $\AA$, 3431-3436, XXXV Encuentro de la Academia Mexicana de Investigación y Docencia en Ingeniería Química, Puerto Vallarta, México 6 al 9 de mayo (2014)

Ramírez-Gómez R.; D. García-Cortés; G. Martínez-de Jesús; M. González-Brambila; A. Alonso y J. Ramírez-Muñoz, Performance evaluation of two high shear impellers in an unbaffled stirred tank, Chem. Eng. Technol.: 38(9), 1519-1529 (2015)

Werner S.; S.C. Kaiser; M. Kraume y D. Eibl, Computational fluid dynamics as a modern tool for engineering characterization of bioreactors, Pharm. Bioprocess: 2(1), 85-99 (2014)

Xie, L.; C.D. Rielly; W. Eagles y G. Ozcan-Taskin, Dispersion of Nano-Particle Clusters Using Mixed Flow and High Shear Impellers in Stirred Tanks, Chem. Eng. Res. Des.: 85(5), 676-684 (2007)

Zalc, J.M; M.M. Alvarez; F.J. Muzzio y B.E. Arik, Extensive Validation of Computed Laminar Flow in a Stirred Tank with Three Rushton Turbines, AIChE J.: 47, 2144-2154 (2001) 